\title{
Application of Oligonucleotide Activation to Restriction Endonuclease NarI
}

\author{
Joseph H. Senesac and Jennifer K. Romanin \\ Promega, Madison, WI, USA
}

BioTechniques 22:1166-1168 (1997)

\begin{abstract}
Restriction endonuclease NarI cleaves DNA using a two-site mechanism, placing it in the Type IIe class of restriction endonucleases. Although these enzymes have very useful recognition sequences, the two-site mechanism limits the practical application. Site preferences often cause incomplete substrate digestion. Oligonucleotide activation of NarI eliminates incomplete digestions, making it possible to use NarI restriction sites in many common molecular biology techniques.

A modified oligonucleotide was chosen for optimal activation of restriction endonuclease NarI. This oligonucleotide was demonstrated to allow complete digestion in many commonly used substrates.
\end{abstract}

\section{INTRODUCTION}

The restriction endonuclease NarI is frequently used in molecular biology experiments. However, some NarI sites in various DNA substrates are refractory to cleavage under standard reaction conditions (4). The kinetics of NarI and other Type IIe systems have been previously described $(4,6)$. Oligonucleotide enhancement of NaeI has demonstrated the usefulness of this method for Type IIe systems in general $(1,2,5)$.

Study of both fast and slow NarI restriction sites in various substrates showed possible activation sequences. Six oligonucleotide sequences (three pairs of sense and antisense) were synthesized and annealed. The oligonucleotides were modified with a phosphorothioate linkage at the cleavage site. This modification allows activation through DNA binding, but prevents endonucleolytic cleavage (2). The three modified double-stranded oligonucleotides were used to develop an activator sequence that allowed complete substrate digestion in all DNAs tested.

\section{MATERIALS AND METHODS}

\section{Digestion Conditions}

All materials, unless otherwise stated below, were obtained in-house from Promega Corporation (Madison, WI, USA). Substrate cleavage was performed at $37^{\circ} \mathrm{C}$ in a $50-\mu \mathrm{L}$ reaction volume containing $50 \mathrm{mM}$ Tris- $\mathrm{HCl}(\mathrm{pH} 8.2$ at $37^{\circ} \mathrm{C}$ ), $5 \mathrm{mM} \mathrm{MgCl}$ and $0.1 \mathrm{mg} / \mathrm{mL}$ acetylated bovine serum albumin. Units of enzyme and digestion time were as indicated. One unit of NarI activity is defined as the amount of NarI needed to completely digest $1 \mu \mathrm{g}$ of Adenovirus-2 (Ad2) DNA at NarI-susceptible sites in $1 \mathrm{~h}$ at $37^{\circ} \mathrm{C}$ in the above reaction mixture (no oligonucleotide activation). All DNA substrates were duplex in form.

\section{Substrate Analysis}

Ad2 and M13mp18 DNAs were obtained from Life Technologies (Gaithersburg, MD, USA), and T7 DNA was obtained from Sigma Chemical (St. Louis, MO, USA). Electrophoresis was performed on $0.7 \%$ agarose slab gels at $90 \mathrm{~V}$, stained with ethidium bromide and photographed using Polaroid 665 film (Cambridge, MA, USA) and a UV transilluminator (Fotodyne, Hartland, WI, USA). After NarI digestion, DNA was purified using the Wizard ${ }^{\mathrm{TM}}$ Minipreps DNA Purification System (Promega) for experiments requiring downstream manipulation of DNA.

\section{Oligonucleotide Synthesis and Annealing}

The synthetic activator oligonucleotides were synthesized using a Model 394 DNA Synthesizer (PE Applied Biosystems, Foster City, CA, USA) using the manufacturer's reagents and protocol. The phosphorothioate linkage was introduced by sulfurization of a phosphite linkage during automated synthesis. The oligonucleotides were purified by reverse-phase HPLC. Peak area evaluation after capillary 
electrophoresis showed the oligonucleotides to be greater than 98\% pure. The duplex oligonucleotide activators were formed by annealing the sense and antisense oligonucleotides.

\section{RESULTS AND DISCUSSION}

Based on current knowledge of NarI site cleavage kinetics (4), three DNA sequences were chosen to evaluate ability to activate cleavage of slow NarI sites. These were sites 1 and 2 from $\phi X 174$ (sense CAAACTGG-S-CGCCGAGCGT and GCTGGTGG-S-CGCCATGTCT, respectively) and site 4 from pBR322 (sense ATTGTAGG-S-CGCCGCCCTA). The site number is based on its location from replication origin. Experiments were performed to optimize cleavage conditions (i.e., the range of activator and enzyme concentration allowing cleavage) and to better understand which sites, and therefore what flanking sequences, best induce cleavage. Of the three sequences, site 2 from $\phi X 174$ showed little activation.

Digests of a number of other substrates showed oligonucleotide 3 ( $\phi$ X174 site 1 as described above) to be the best activating sequence. The concentration of oligonucleotide necessary for complete digestion was optimal at 0.4

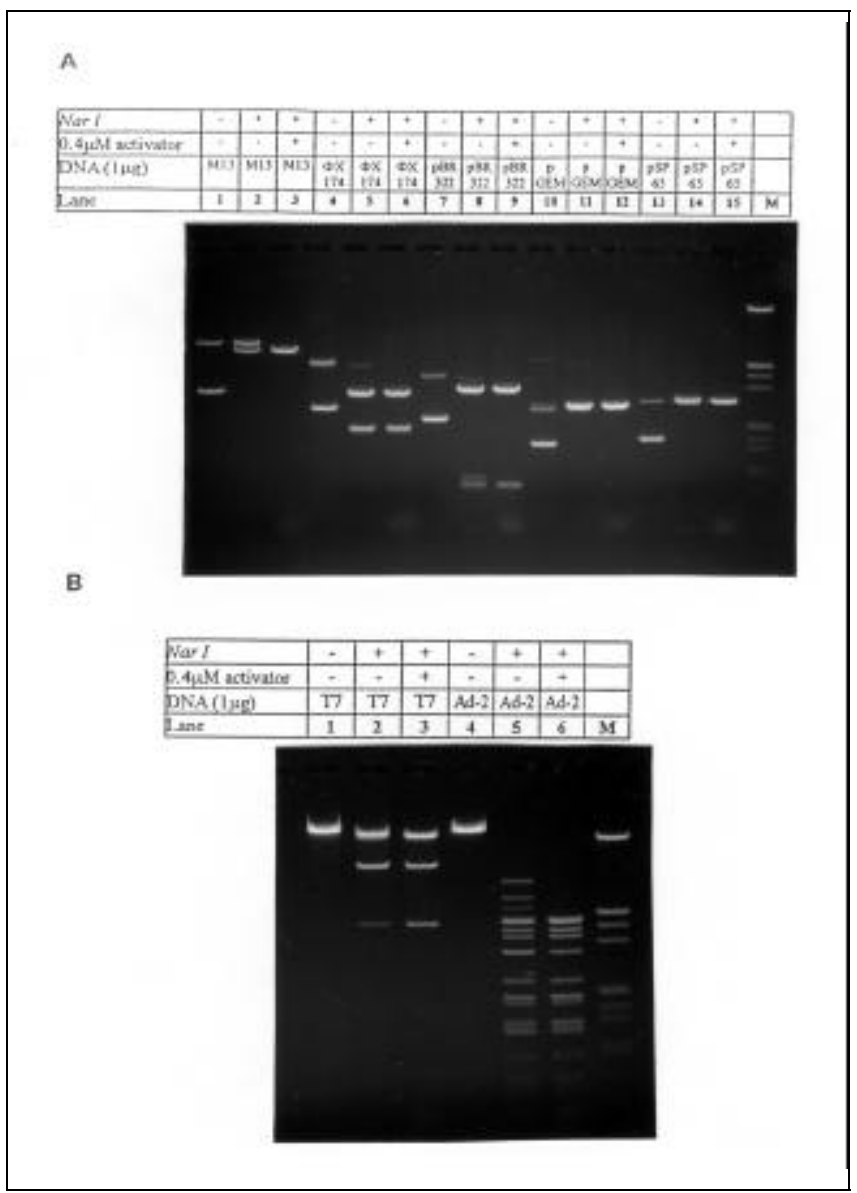

Figure 1. Analysis of NarI cleavage of various substrate DNA with and without activator. (A) Lane M: lambda HindIII/EcoRI markers; lanes 1, 4, 7, 10 and 13: $1 \mu \mathrm{g}$ each of M13mp18, $\phi$ X174, pBR322, pGEM $^{\circledR}$ and pSP65 DNA, respectively, incubated for 60 min with $12 \mathrm{U}$ NarI; lanes 2, 5, 8, 11 and 14: the same as above but with $0.4 \mu \mathrm{M}$ activator; lanes 3, 6, 9, 12 and 15: $1 \mu \mathrm{g}$ of uncut substrate as indicated. (B) Lane M: lambda HindIII/EcoRI markers; lanes 2 and 5: $1 \mu \mathrm{g}$ each of T7 and Ad2 DNA, respectively, incubated for 60 min with $2 \mathrm{U}$ NarI; lanes 3 and 6: the same as lanes 2 and 5 but with $0.4 \mu \mathrm{M}$ activator; lanes 1 and $4: 1 \mu \mathrm{g}$ of uncut substrate as indicated. 
$\mu \mathrm{M}$. Sequences offering greater activation tended to show the effect at lower activator oligonucleotide concentrations than those offering less activation because of a rank order of sites (4). The range of oligonucleotide concentration providing activation was wider than the other sites, allowing a more robust reaction against operator error. In all substrates studied, a complete digest was obtained under suitable reaction conditions (Figure 1).

Published literature indicates that there are two slow NarI sites in pBR322 DNA (3). Experiments using pBR322 demonstrated that the digestion difficulty may be related to the physical distribution of the slow NarI sites, rather than the kinetics that would result from their flanking sequences. Type IIe enzymes, even if given two kinetically feasible sites, may require a certain physical distance between these sites to allow binding to both the activation and catalytic sites on the enzyme. The banding pattern of an incomplete digest on pBR322 does not necessarily suggest the presence of slow site(s), but rather a mixture of digests terminating at a site that might be difficult to activate in a cis configuration by the other remaining uncut site(s). As an activator oligonucleotide will quickly allow digestion of these sites, it could be argued that the site locations, not site preferences, cause the incomplete digestion of $\mathrm{pBR} 322$.

Further experiments (data not shown) have shown that the activator chosen will not interfere, if properly purified, with a cut-ligate-recut experiment.

We have shown that the activation of NarI results in greater cleavage efficiency without interfering in downstream applications. This is a desirable result for efficient completion of work in the molecular biology laboratory. Activation reactions are not subject to long digestion times or incomplete digests. It is anticipated that other Type IIe restriction endonucleases (i.e., SacII, EcoRII) may benefit from development of similar activator oligonucleotides. The activator oligonucleotide described in this manuscript is now available from Promega as a result of this research.

\section{REFERENCES}

1.Conrad, M. and M. Topal. 1989. DNA and spermidine provide a switch mechanism to regulate the activity of restriction enzyme NaeI. Proc. Natl. Acad. Sci. USA 86:9707-9711.

2.Conrad, M. and M. Topal. 1992. Modified DNA fragments activate NaeI cleavage of refractory DNA sites. Nucleic Acids Res. 20:5127-5130.

3.New England Biolabs Catalog. 1995. Site Preferences, p. 212. Beverly, MA.

4.Oller, A., W. Vanden Broek, M. Conrad and M. Topal. 1991. Ability of DNA and spermidine to affect the activity of restriction endonucleases from several bacterial species. Biochemistry 30:2543-2549.

5.Senesac, J.H. and J.R. Allen. 1995. Oligonucleotide activation of the Type IIe restriction enzyme NaeI for digestion of refractory sites. BioTechniques 19:990-993.

6.Yang, C.C. and M. Topal. 1992. Nonidentical DNA-binding sites of endonuclease NaeI recognize different families of sequences flanking the recognition site. Biochemistry 31:9657-9664.

Address correspondence to Jennifer K. Romanin, Promega Corporation, 2800 Woods Hollow Road, Madison, WI 53711 5399, USA. Internet:jromanin@promega.com 